\title{
Modyular na pag-aaral sa aspekto ng pag-uugali ng mga mag-aaral sa kolehiyo
}

Jurado, Rhea Mae

Veritas College of Irosin, Philippines (riyamaejurado@gmail.com)

Belen Joshua

Veritas College of Irosin, Philippines (joshuafragabelen@gmail.com)

Mateo, Rica

Veritas College of Irosin, Philippines (mateorica97@gmail.com)

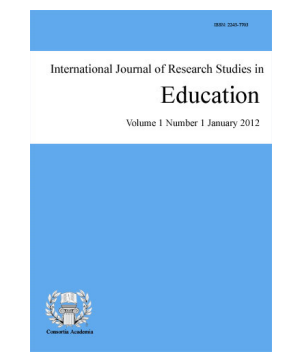

ISSN: 2243-7703 Online ISSN: 2243-7711

OPEN ACCESS

Gelilio, Eric $\bowtie$

Veritas College of Irosin, Philippines (gelilioeric@gmail.com)

Sorsogon State University, Philippines

Gallanosa National High School, Philippines

Ginaga, Ana Marie

Veritas College of Irosin, Philippines

Received: 15 January 2022

Available Online: 23 January 2022

\section{Abstract}

Amidst of the pandemic, teaching and learning were conducted through Modular Learning. As such, students are responsible for completing and submitting school activities at their own time and location. The purpose of the study was to identify and evaluate the impacts of the Modular Learning on the behavioral aspects of Veritans in the first year of the College of Commerce in the year 2021-2022. Descriptive analysis was the design used in the research. Random sampling was used to collect data from 150 students participated from first year of the College of Commerce. The findings were categorized according to the psychological changes of the students as well as the interests and personalities of the college students while using the Modular Learning. According to Smart and Cappel's (2006), Students who were interested in information or those are familiar with it showed a higher level of interest. Further, the loss of interest and focus may explain its negative impact on students 'behavior. According to a study conducted by Armstrong-Mensahet al. (2020), more than half $(53.6 \%)$ of the participants reported that they remained motivated and successfully completed their assignments on time. (3.4\%) reported that they had trouble motivating themselves to learn. More than $50 \%$ of the students surveyed did not miss to $\log$ in to take scheduled course exams and $47.5 \%$ interacted with their peers on group course assignments. Less than half $(45.1 \%)$ of the students said they were able to take the time to focus and do their schoolwork.

Keywords: modular learning, student's behavior, impacts, tertiary education, effective practices 


\section{Modyular na pag-aaral sa aspekto ng pag-uugali ng mga mag-aaral sa kolehiyo}

\section{Introduction}

Sinubok ng pandemya ang sistema ng edukasyon sa Pilipinas na nagresulta sa libu-libong mag-aaral na apektado ng "new normal" na edukasyon, isa sa mga pinaka-matinding naapektuhan nito ay ang mga mag-aaral sa kolehiyo. Dahil dito, ipinatupad ng Kagawaran ng Edukasyon at ng Commission on Higher Education ang iba't ibang mga opsyon sa paghahatid ng pagkatuto na maaaring gamitin, kabilang dito ang Limited to Blended Learning at Distance Learning upang matiyak ang kaligtasan ng mga mag-aaral mula elementarya hanggang kolehiyo at maging ang mga guro. Ang Modyular na pag-aaral ay isa sa mga opsyon na kung saan layunin nitong bigyan ng tungkulin ang bawat mga estudyante na sagutin at ipasa sa takdang oras ang mga modyuls sa pamamagitan ng paggamit ng mga mag-aaral ng Self-Learning Modules o SLM.

Ayon kay Secretary Leonor Briones dekada ng isinasagawa ang Modyular na pag-aaral pati na rin ang Blended Learning. Sa pinakamahabang panahon, ang Unibersidad ng Pilipinas ay nakagawa at nagdadalubhasa sa distance education, at ang mga mag-aaral ng edukasyon ay pamilyar na rito. Dahil na rin sa potensyal na panganib sa kalusugan ng COVID19, napilitang lumipat ang mga estudyante mula sa pisikal na pagpasok sa mga paaralan sa kolehiyo tungo sa pag-angkop sa bagong paraan ng edukasyon. Dahil dito maaari itong magdulot ng epekto sa sikolohikal, interes, at pagkatao ng mga estudyante dahil sa pagkawala ng tradisyunal na pamamaraan ng edukasyon. Ayon kay Mojtabai et al.,(2015); Richardson, (2015), Ang mga mag-aaral na nahaharap sa mga problema sa kalusugang pang-kaisipan ay may malaking posibilidad na hindi matapos o mapasa ang mga modyuls na kailangang isumite sa tinakdang oras dahil sa kawalan ng disiplina sa sarili, motibasyon, at pokus na nagdudulot ng malaking posibilidad na mag-drop out sa Unibersidad at maaaring makakuha ng mababang marka.

Sambit kay Moos (1980) Ang kahalagahan ng pisikal at panlipunang kapaligiran, tulad ng mga instruktor, mag-aaral, paaralan, institusyon, at iba pang pampublikong espasyo, ay sinisiyasat kaugnay ng pakikipag-ugnayan ng tao-kapaligiran. Upang masuri ang mga natatanging hamon na kinakaharap ng mga indibidwal na mag-aaral at kung paano magdisenyo ng mga estratehiya upang gawing mas komportable at kaaya-aya ang kapaligiran ng pag-aaral. Alinsunod sa pag-aaral na isinagawa ni Osman (2005), 70\% ng mga mag-aaral ang nakadama ng mas komportableng pagsali sa isang online na forum ng talakayan kumpara sa isang talakayan sa klase. Ang mga pagkakaibang ito sa mga saloobin tungkol sa mga Discussion Board ay maaaring maiugnay sa mga pagkakaiba ng personalidad ng bawat mag-aaral, kung saan ang isang introvert na mag-aaral ay maaaring nakakaramdam ng komportable o kasiyahan sa pakiramdam ng pagkakaroon ng kunpidensyal at privacy na nalikha sa pamamagitan ng paglahok sa isang birtual na talakayan kumpara sa isang harapang talakayan.

Banggit sa pag-aaral ni Felix (2001) napag-alaman niya na ang mga mag-aaral ay may negatibong saloobin sa Online Learning kapag walang sapat na personal na komunikasyon o pakikipag-ugnayan sa kanilang mga instruktors. Ayon sa resulta ng pag-aaral na ito, 10 ang bilang ng mga mag-aaral ang nababahala sa kakulangan sa gabay ng isang guro kumpara sa 3 mga mag-aaral na nadama ang eksaktong kabaligtaran. Dagdag pa ni Lee, Srinivasan, Trail, Lewis and Lopez (2011) sa kanilang pag-aaral, napag-alaman na pinahahalagahan at mas kapaki-pakinabang para sa mga mag-aaral ang pakikipag-ugnayan sa mga instruktor at mga kasamahan nila. Dahil dito natutuhan na ang pangunahing solusyon ay ang pagkakaroon ng pakikipag-ugnayan sa mga guro at maging sa mga kasamahan. Iminungkahi rin nila Lee et al. ang Online o ang On-Campus Study Group bilang isang paraan upang maiwasan ang pagkakaroon ng pakiramdam na nag-iisa at hindi pagiging komportable sa pag-aaral ng mga mag-aaral dahil lamang sa kakulangan ng pakikipag-ugnayan.

Alinsunod sa talakayan sa itaas, ang layunin ng pananaliksik na ito ay alamin ang mga naging impak ng 
modyular na pag-aaral sa aspekto ng pag-uugali ng mga Veritans sa unang taon ng kolehiyo sa kursong Komersyo. Gayundin, ang pag-aaral na ito ay naglalayon na alamin ang mga pamamaraan o solusyon at magbibigay rin ng kaalaman sa mga mag-aaral, magulang at guro na nahihirapan sa mga bagong pamamaraan ng pagkatuto sa gitna ng pandemya.

\subsection{Paglalahad ng suliranin}

Ang pananalisik na ito ay nakatuon sa pangunahing layunin nito na kung saan ay matukoy ang mga naging impak ng Modyular na pag-aaral sa aspekto ng pag-uugali ng mga Veritans sa unang taon sa kurso ng komersyo. Nilalayon nitong sagutin nang tiyak ang mga sumusunod na katanungan:

Ano-ano ang naging impak ng Modyular na pag-aaral sa oras ng paggamit ng internet ng mga Veritans sa Komersyo?

$>\quad$ Ano-ano ang mga salik na nakakaapekto sa Modyular na pag-aaral sa pag-uugali ng mga Viretans sa Komersyo? Sikolohikal, interes, at pagkatao.

$>\quad$ May makabuluhang pagkakatulad ba ang impak ng Modyular na pag-aaral sa pagbabago ng pag-uugali ng mga Viretans sa Komersyo?

\section{Pamaraang ginamit}

Ginamit ang deskriptibong pamamaraan ng pananaliksik upang matukoy ang impak ng Modyular na pag-aaral sa aspekto ng pag-uugali ng isang mag-aaral sa kolehiyo. Random sampling ang ginamit ng mga mananaliksik sa 150 na napiling kalahok mula sa pangkat ng mga mag-aaral sa unang taon sa kolehiyo na naka-enrol ng kursong Komersyo sa Veritas College Irosin, Sorsogon. Isinagawa ang pagsisiyasat sa pamamagitan ng online na survey upang mas mapadali ang pakikipag-ugnayan sa mga mag-aaral na kalahok sa pananaliksik na hindi na kinakailangan pa ng harapang panayam at upang masunod na rin ang mga protokols sa pangkalusugan dahil sa kinakaharap na pandemya. Ang mga mananaliksik ay gumamit ng tseklist na talatanungan sa pangangalap ng datos. Ang mga mananaliksik ay lumikha ng sariling gawang online na talatanungan na magsisilbing instrumento sa pananaliksik. Messenger App ang pangunahing gamit upang makakalap ng sagot mula sa napiling mga kalahok. Ginamit ng mananaliksik ang sariling gawang palatanungan patungkol sa dependent at independent variables na pangunahing mapagkukunan at instrumento sa pagkolekta at paghambing ng mga kinakailangang datos na may kaugnayan sa pag-aaral. Ang mga tugon ay itinala bilang batayan para sa istatistikong pagsusuri ng mga datos. Ayon kay Mbahi (2001), Ang deskriptibo at sarbey na disenyo sa pananaliksik ay nakatuon sa pangangalap ng datos upang ilarawan at suriin ang mga kasalukuyang sitwasyon, umiiral na gawi, paniniwala, saloobin, at patuloy na proseso sa iba pang mga bagay.

\section{Mga natuklasan}

3.1 Impak ng Modyular na pag-aaral sa oras ng paggamit ng internet ng mga Veritans sa Komersyo.

\section{Talahayan 1}

Oras na Ginugugol sa Paggamit ng Internet ng mga Mag-aaral sa Kolehiyo.

\begin{tabular}{lcc}
\hline \multicolumn{1}{c}{ Oras na Ginugol ng Mga } & Bilang ng Mag-aaral & Rango \\
\hline Sa loob ng 10 oras & 20 & 5 \\
Sa loob ng 5 oras & 25 & 3 \\
Sa loob ng 3 oras & 43 & 1 \\
Sa loob ng 1-2 oras & 41 & 2 \\
Kumulang sa isang oras & 21 & 4 \\
\hline
\end{tabular}

Batay sa talahanayan, ang pinakamataas na oras na ginugol ng isang mag-aaral sa unang taon ng Komersyo 
Jurado, R. M., Belen, J., Mateo, R., Gelilio, E., \& Ginaga, A. M.

sa kolehiyo ng Veritas Irosin sa bawat araw ay nasa loob ng 3 oras, na sinusundan ng 41 na mga mag-aaral na naglaan ng 1-2 oras sa paggamit ng internet, 25 na mga mag-aaral na gumamit ng internet sa loob ng 5 oras, 21 na mag-aaral na nagkunsumo ng internet nang wala pang 1 oras at 20 na mag-aaral na gumamit ng internet sa loob ng 10 oras bawat araw. Ayon kay Foerster, Milena et al. (2019) natuklasan sa kanilang pag-aaral na ang labis na paggamit ng internet ay maaaring humantong sa mga sintomas ng insomnia sa mga kabataan. Ang mga kabataan na gumamit ng internet nang higit pa sa 3 oras bawat araw ay may epekto sa kanilang oras ng pagtulog kaysa sa mga hindi gaanong gumagamit nito. Batay sa naging resulta, naging malinaw na ang paglipat sa modyular na pag-aaral ay nagpataas ng pagkababad ng mga mag-aaral sa internet, kumpara sa 1-2 oras bawat araw na karaniwang ginugugol ng mga mag-aaral sa internet kapag sila ay nasa isang tradisyonal na edukasyon (Does my internet usage affect my health. 2020).

\subsection{Salik na nakakaapekto sa Modyular na pag-aaral sa pag-uugali ng mga Viretans sa Komersyo}

\section{Talahayan 2}

Mga Salik na nakakaapekto sa Modyular na pag-aaral sa Sikolohikal na aspekto ng Pag-uugali

\begin{tabular}{|c|c|c|c|}
\hline \multirow{2}{*}{$\begin{array}{l}\text { Sikolohikal na Aspekto ng mga } \\
\text { Mag-aaral }\end{array}$} & \multicolumn{3}{|c|}{ Bahagdan } \\
\hline & Oo & Hindi & Wala pang naging pasya \\
\hline $\begin{array}{l}\text { Nawawalan ka ba ng motibasyon o pokus na } \\
\text { masagutan ang iyong mga aralin? }\end{array}$ & 59.3 & 29.3 & 11.3 \\
\hline $\begin{array}{l}\text { Naging mainitin ba ang iyong ulo o } \\
\text { madaling magalit simula ng umangkop ka sa } \\
\text { Modyular na pagkatuto? }\end{array}$ & 46 & 38 & 16 \\
\hline $\begin{array}{l}\text { Nakakaramdam kaba ng pagod o stress } \\
\text { habang ikaw ay sumasagot ng iyong mga } \\
\text { modyuls? }\end{array}$ & 83.3 & 10.7 & 6 \\
\hline Kabuoan & 62.9 & 26 & 11.1 \\
\hline
\end{tabular}

Ayon sa resulta na nakabatay sa talahanayan sa itaas, napag-alaman na $83.3 \%$ na bilang ng mga mag- aaral ang nakakaramdam ng pagod o stress habang sila ay sumasagot ng kanilang mga modyuls. Mas mataas ang datos nito kumpara sa mga mag-aaral na nakakaramdam ng pagkawala ng motibasyon o pokus sa pagsagot ng kanilang mga modyuls na may bilang ng 59.3\%, habang ang mga mag-aaral na nakararanas naman ng pagiging mainitin ng ulo o ang madaling makaramdam ng galit ay may bilang naman na 46\%. Nagpapakita lamang ito na ang modyular na pag-aaral ay nagresulta sa negatibong epekto sa sikolohikal na aspekto ng mga mag-aaral sa unang taon ng Komersyo sa kolehiyo ng Veritas Irosin. Sa kabilang dako, ay nakakuha naman ng 38\% na bilang ng mga mag-aaral ang nagsabing hindi sila nakararanas ng pagiging mainitin ang ulo o ang madaling makaramdam ng galit. Nagpapahayag lamang ito na mas maliit ang pagkakataon nito na magkaroon ng impak sa sikolohikal na aspekto ng mga mag-aaral sa unang taon ng Komersyo sa kolehiyo ng Viretas Irosin na umaangkop sa modyular na pag-aaral.

\section{Talahanayan 3}

Mga Salik na nakakaapekto sa Modyular na pag-aaral sa Interes na aspekto ng Pag-uugali

\begin{tabular}{|c|c|c|c|}
\hline \multirow{2}{*}{$\begin{array}{c}\text { Interes na Aspekto ng mga } \\
\text { Mag-aaral }\end{array}$} & \multicolumn{3}{|c|}{ Bahagdan } \\
\hline & Oo & Hindi & Wala pang naging pasya \\
\hline $\begin{array}{l}\text { Nagagawa mo pa bang gawin ang iyong mga } \\
\text { libangan? Hal. Ay maglaro ng video game, } \\
\text { gumuhit, magbasa atbp. habang ikaw ay nasa } \\
\text { modyular na pag-aaral? }\end{array}$ & 62.7 & 31.3 & 6 \\
\hline $\begin{array}{l}\text { Nagkaroon ka pa ba ng oras lumabas at } \\
\text { makasama ang iyong mga kaibigan at } \\
\text { pamilya kahit na ikaw ay nasa modyular na } \\
\text { pag-aaral? }\end{array}$ & 76 & 16 & 8 \\
\hline $\begin{array}{l}\text { Naba-balanse mo ba ang iyong oras sa } \\
\text { pag-aaral at ang iyong mga personal na } \\
\text { gawain? }\end{array}$ & 48 & 27.3 & 19.3 \\
\hline Kabuoan & 62.2 & 24.8 & 11.1 \\
\hline
\end{tabular}


Modyular na pag-aaral sa aspekto ng pag-uugali ng mga mag-aaral sa kolehiyo

Lumabas sa naging resulta na $31.3 \%$ ang bilang ng mga mag-aaral na nagsasabing hindi na nila nagagawa ang kani-kanilang mga libangan sa kadahilanang wala na silang sapat na oras na pwede nilang mailaan ukol dito. Ngunit kabaligtaran naman ito sa naging datos na lumabas na $76 \%$ ang bilang ng mga mag-aaral na nagsasabing nakakapag-laan pa sila ng oras na lumabas at makasama ang kani-kanilang mga kaibigan at pamilya sa kabila ng umiiral na modyular na pag-aaral. Sa kabuuan $62.2 \%$ ng mga mag-aaral ang nagsabing nagagawa pa nilang gawin ang kanilang mga interes o mga libangan habang sila ay umaangkop sa bagong sistema ng pagkatuto. Nagpapakita lamang ito na naging positibo ang epekto ng modyular na pag-aaral sa oras ng mga mag-aaral dahil nagagawa pa nila ang mga aktibidad na nagpapasaya sa kanila habang ginagampanan nila ang mga responsibilidad nila bilang mga mag-aaral sa kolehiyo.

\section{Talahanayan 4}

Mga Salik na nakakaapekto sa Modyular na pag-aaral sa Pagkatao na aspekto ng Pag-uugali

\begin{tabular}{lccc}
\hline \multicolumn{1}{c}{$\begin{array}{c}\text { Pagkatao na Aspekto ng mga } \\
\text { Mag-aaral }\end{array}$} & Oo & Hahagdan & Wala pang naging pasya \\
\cline { 2 - 4 } $\begin{array}{l}\text { Mas komportable o nasisiyahan ka bang } \\
\text { matuto sa pamamaraan ng modyular na pag } \\
\text { aaral kaysa sa tradisyonal na pagkatuto? }\end{array}$ & 16 & 71.1 & 12.7 \\
$\begin{array}{l}\text { Mas nagkaroon kaba ng maraming kaibigan } \\
\text { ngayong modyular na pag-aaral kompara sa } \\
\text { dating tradisyonal na pag-aaral? }\end{array}$ & 20 & 64.7 & 15.3 \\
$\begin{array}{l}\text { Mas naging produktibo kaba ngayong } \\
\text { modyular na pag-aaral kompara sa } \\
\text { tradisyonal na pag-aaral? }\end{array}$ & 20.7 & 63.3 & 16 \\
$\begin{array}{l}\text { Sa iyong palagay nalinang ba ang iyong } \\
\text { pakikipag kommunikasyon sa ibang tao at } \\
\text { ang paggawa ng sariling desisyon habang }\end{array}$ & 34.7 & 50 & 15.3 \\
$\begin{array}{l}\text { ikaw ay umaangkop sa modyular na pag } \\
\text { aaral? Kabuoan }\end{array}$ & & & \\
\hline
\end{tabular}

Batay sa naging resulta sa talahanayan, nakakuha ng $71.1 \%$ ang bilang ng hindi naging komportable o nasisiyahan na mga mag-aaral sa modyular na pag-aaral na nagresulta upang mabawasan ang tiwala nila sa kanilang sarili na sila ay natuto sa pamamagitan ng makabagong pagkatuto sa gitna ng pandemya. Nakakuha naman ng 64.7\% na bilang ng mga mag-aaral ang nagsabing nabawasan o hindi sila nagkaroon ng pagkakataong magkaroon ng bagong kaibigan dahil narin sa pagkawala ng tradisyonal na pag-aaral na nagbibigay ng pagkakataon upang magkaroon ng pisikal na pakikisalamuha sa ibang tao. $34.7 \%$ naman ang nagsabing nalinang nila o mas napayabong nila ang kanilang kakayahan sa pakikipag komunikasyon sa iba dahil narin sa nagkaroon sila ng daan upang makipagkomunikasyon sa ibang tao gamit ang mga online na app.

Ayon kay Brooks et al. (2020). mas marami ang naging negatibong epekto ng modyular na pag aaral para sa mga mag-aaral dahil sa pagkawala ng pisikal na pakikipag-ugnayan sa iba dahil sa umiiral na COVID19, lalo na sa mga kapwa nilang mag-aaral, humantong ito sa mga negatibong emosyon tulad ng pagkabagot at pagkabalisa ng ilan sa mga mag-aaral sa mga unibersidad. Higit pa rito, ang mga pagsasara ng paaralan ay may epekto rin sa mga komunidad, na nagreresulta sa pagtaas ng pangangailangan para sa mga kagamitan at internet, pati na rin ang pagkabalisa sa pag-iisip para sa mga magulang ng bawat isang mag-aaral (Daniel, 2020). Nagpapatunay ito sa pag-aaral na $83 \%$ ang bilang ng mga hindi sang-ayon sa pagiging epektibo ng makabagong sistema ng pagkatuto sa gitna ng kinakaharap nating pandemya.

\subsection{Kabuluhang pagkakatulad sa impakng Modyular na pag-aaral sa pagbabago ng pag-uugali ng mga}

\section{Viretans sa Komersyo}

Nagkaroon ng makabuluhang impak ang Modyular na pag-aaral sa pagbabago ng pag-uugali ng mga Viretans sa Komersyo dahil naging dahilan ito ng pagkakaroon ng mababang kalidad ng edukasyon. Maraming nahihirapang mga mag-aaral lalong lalo na ang mga mga-aaral sa kolehiyo na unawain ang mga komplikadong 
Jurado, R. M., Belen, J., Mateo, R., Gelilio, E., \& Ginaga, A. M.

aralin na ibinigay ng kanilang mga guro sa kadahilanang walang sapat na pagtuturo o komunikasyong nagaganap sa pagitan ng mga guro at ng mga mag-aaral. Naging resulta rin ito sa pagkakaroon ng negatibong mga emosyon at kilos ng bawat mag-aaral habang sila ay umaangkop sa Modyular na pag-aaral.

\section{Kongklusyon}

Batay sa natuklasan, nabuo ang sumusunod na kongklusyon:

Nagkaroon ng impak ang oras ng paggamit ng internet sa pag-uugali ng 150 na mga mag-aaral mula sa unang taon sa kursong Komersyo ng Veritas College. Sapagkat ang mga mag-aaral na labis ang oras ng paggugol sa internet ay maaaring makaranas ng negatibong epekto lalo na sa kanilang kalusugan, maari itong maging sanhi na makaranas ang mga mag-aaral ng kakulangan sa tulog na maaring magdulot upang hindi sila maging produktibo at magkaroon ng negatibong impak sa kanilang pag-aaral. Tumaas din ang bilang ng mga mag-aaral ang nababad sa internet habang nasa modyular na pag-aaral dahil ito ang isa sa mga naging katuwang ng mga mag-aaral sa pagsagot ng kanilang mga aralin. Mataas ito kumpara sa oras na kinu-konsumo nila noong nasa tradisyonal na pag-aaral pa sila.

Dahil nasa Modyular na pag-aaral ay naging hawak ng mga mag-aaral ang kani-kanilang mga oras, ibig sabihin lamang nito ay nagkaroon sila ng pagkakataon na mabalanse ang kanilang paggawa sa kanilang mga aktibidad maliban sa pag-aaral tulad na lamang ng kanilang mga libangan. Kumpara noong nasa tradisyonal na pag-aaral pa ay limitado at nakatutok lamang sila sa kanilang pag-aaral ngunit dahil sa paglipat sa modyular na pag-aaral ay nagkaroon sila ng kalayaan upang maglaan ng oras sa iba pang mga pansariling gawain.

Nagkaroon ng negatibong epekto ang Modyular na pag-aaral sa pagkatuto ng mga mag-aaral dahil sa kakulangan ng gabay o komunikasyon mula sa kanilang mga guro upang maunawaan ang bawat aralin na kailangan nilang pag-aralan. Nakakaramdam sila ng pagkawala ng motibasyon, pokus upang matuto, pagkalito, pagkabalisa at stress na nagiging sanhi upang bumaba ang pagiging produktibo nila at mag resulta upang hindi maging komportable o hindi kontento ang mga mag-aaral sa bagong pamamaraan ng pagkatuto.

Napag-alaman din na nagkaroon ng impak ang Modyular na pag-aaral sa buhay panlipunan ng mga mag-aaral. Dahil sa kinakaharap nating pandemya pinagbawal ang pagpunta ng mga mag-aaral sa mga paaralan na kung saan nawalan sila ng pagkakataon na makasalamuha o makakilala ng bagong kaibigan. Dahil dito nagkaroon ng balakid ang pakikipagkomunikasyon nila mula sa ibang tao na maaring magresulta sa negatibong epekto sa sikolohikal at pagkatao ng mga mag-aaral at maging dahilan upang magkaroon sila ng mababang kumpiyansa sa desisyon at sarili.

\subsection{Rekomendasyon}

Sa kabuuan, mas marami ang naging negatibong impak ng Modyular na pag-aaral sa aspekto ng sikolohikal, interes, at pagkatao ng mga mag-aaral sa unang taon sa kursong Komersyo ng Veritas College. Batay sa mga resulta at kongklusyon ng pag-aaral na ito, ang sumusunod na mga rekomendasyon ay nabuo:

Gawing kapani-pakinabang ang bawat oras. Maglaan ng iskedyul sa bawat gawaing kailangan nilang tapusin katulad na lamang ng pag-aaral at iba pang mga pansariling aktibidad ng bawat mag-aaral sa kolehiyo na naka-enrol sa kursong Komersyo upang mabalanse at matiyak na nagawa ang mga aktibidad ng hindi nakakaranas ng pagkalito o pagkakaroon ng stress. Upang magawa ito ng matagumpay, kinakailangan din ng gabay mula sa kanilang mga guro sa pamamagitan ng iba't ibang pamamaraan ng birtwal na komunikasyon halimbawa ay Messenger, Zoom, o Google Meet ng sa ganoon ay masubaybayan ng mga guro ang kalagayan ng mga mag-aaral habang sila ay nasa Modyular na pag-aaral.

Magsagawa ng Online na forum upang malaman ng mga guro ang istado o lebel ng mga natutuhan ng bawat mga mag-aaral sa Kolehiyo halimbawa na lamang nito ay ang pagkakaroon ng online na pagsusulit, pagtatasa at ilan pang mga aktibidad sa pag-aaral upang maging batayan ng mga guro sa kung hanggang saan ang natutuhan

34 Consortia Academia Publishing (A partner of Network of Professional Researchers and Educators) 
Modyular na pag-aaral sa aspekto ng pag-uugali ng mga mag-aaral sa kolehiyo

ng mga mag-aaral. Mahalagang hakbang ito upang maiwasan ng mga mag-aaral na makaranas ng pagkalito at stress sa pagsagot ng mga modyuls at magagawa nitong maging produktibo ang mga mag-aaral.

Magkaroon ng pagpapabuti sa disenyo ng paggawa ng mga modyuls upang mahikayat nito ang mga mag-aaral na basahin at pag-aralan ang mga ito. Mahalaga ito upang magkaroon ng pokus at mas maging madali para sa mga mag-aaral na maunawaan ang mga nilalaman ng mga modyuls kapag ito ay sinuri at maayos ang pagkakabalangkas nito.

Magpatupad ng birtwal na guidance counseling na maaring isagawa tuwing katapusan ng buwan upang masubaybayan at gabayan ang mga mag-aaral sa kolehiyo lalong lalo na sa pagpapaunlad ng kanilang pag-uugali habang sila ay nasa Modyular na pag-aaral. Makakatulong ang pagkakaroon ng konsultasyon mula sa paaralan upang maiwasan ang pagtaas ng kaso ng mga mag-aaral na nakararanas ng stress, pagkawala ng motibasyon sa pag-aaral at iba pang mga negatibong epekto ng modyular na pag-aaral sa kanilang sikolohikal, interes at pagkatao. Makakatulong rin ito upang magkaroon ng kaalaman ang mga magulang ng bawat mag-aaral sa kung paano nila bibigyan ng patnubay ang kanilang mga anak habang sila ay nag-aaral sa kani-kanilang mga tahanan dahil sa kasalukuyang pandemya at sa makabagong pamamaraan ng pagkatuto, ang kanilang mga tahanan ang nagsisilbing silid-aralan nila at ang mga magulang ang tanging makakatulong sa kanila upang maging epektibo ang modyular na pag-aaral ng walang negatibong epekto sa pag-uugali ng mga mag-aaral.

\section{Talasanggunian}

Armstrong-Mensah, E., Ramsey-White, K., Yankey, B., \& Self-Brown, S. (2020). COVID-19 and Distance Learning: Effects on Georgia State University School of Public Health Students. Front. Public Health 8 , 576227. https://doi.org/10.3389/fpubh.2020.576227

Brooks, S. K., Webster, R. K., Smith, L. E., Woodland, L., Wessely, S., Greenberg, N., \& Rubin, G. J. (2020). The psychological impact of quarantine and how to reduce it: Rapid review of the evidence. The Lancet, 395(10227), 912-920. https://doi.org/10.1016/S0140-6736(20)304608

Brown, P. (2016). The invisible problem? Improving students' mental health. Higher Education Policy Institute, 88, 66. https://www.hepi.ac.uk/2016/09/22/3592/

Daniel, S. J. (2020). Education and the COVID-19 pandemic. Prospects, 49(12), 9196. https://doi.org/10.1007/s11125-020-09464-3

Does my internet usage affect my health? IMedicine or Malarkey. (2020, February 14). CareSpot Family of Urgent Care Brands. https://www.carespot.com/blog/does-internet-usage-affect-your-health/

Epekto Ng Modyular Na Modalidad Ng Pagtuturo Sa Akademikong Pagganap. (n.d.). Scribd. https://www.scribd.com/document/531594085/Epekto-Ng-Modyular-Na-Modalidad-Ng-Pagtuturo-SaAkademikong-Pagganap

Felix, U. (2001). A multivariate analysis of students' experience of web based learning. Australian Journal of Educational Technology, 17(1), 21-36.

Foerster, M., Henneke, A., Chetty-Mhlanga, S., \& Röösli, M. (2019). Impact of Adolescents' Screen Time and Nocturnal Mobile Phone-Related Awakenings on Sleep and General Health Symptoms: A Prospective Cohort Study. International journal of environmental research and public health, 16(3), 518. https://doi.org/10.3390/ijerph16030518

Lee, S.J., Srinivasan, S., Trail, T., Lewis, D., \& Lopez, S. (2011). Examining the relationship among student perception of support, course satisfaction, and learning outcomes in online learning. The Internet and Higher Education. https://doi.org/10.1016/j.iheduc.2011.04.001

Mojtabai, R., Stuart, E. A., Hwang, I., Eaton, W. W., Sampson, N., \& Kessler, R. C. (2015). Long-term effects of mental disorders on educational attainment in the National Comorbidity Survey ten-year follow-up. Social Psychiatry and Psychiatric Epidemiology, 50(10), 1577-1591. https://doi.org/10.1007/s00127-015-10835

Moos, R. H. (1980). Evaluating classroom learning environments. Studies in Educational Evaluation, 6(3), 239-252. https://doi.org/10.1016/0191-491X(80)90027-9 
Jurado, R. M., Belen, J., Mateo, R., Gelilio, E., \& Ginaga, A. M.

Osman, M. E. (2005). Students' reaction to WebCT: Implications for designing online learning environments. International Journal of Instructional Media, 32(4), 353-362.

Richardson, J. T. E. (2015). Academic attainment in students with mental health difficulties in distance education. International Journal of Mental Health, 44(3), 231-240. https://doi.org/10.1080/00207411.2015.1035084

Smart, K. L., \& Cappel, J. J. (2006). Students' perceptions of online learning: A comparative study. Journal of Information Technology Education, 5(1), 201-219. 